\title{
BMJ Open Sex-specific and age-specific characteristics of body composition and its effect on bone mineral density in adults in southern China: a cross- sectional study
}

\author{
Zeyu Xiao (D) , 1,2 Zhiqiang Tan,, ${ }^{2,3}$ Jingjie Shang, ${ }^{2,3}$ Yong Cheng, ${ }^{2,3}$ Yongjin Tang, ${ }^{2,3}$ \\ Bin Guo, ${ }^{2,3}$ Jian Gong, ${ }^{2,3}$ Hao Xu $u^{2,3}$
}

To cite: Xiao Z, Tan Z, Shang J, et al. Sex-specific and agespecific characteristics of body composition and its effect on bone mineral density in adults in southern China: a crosssectional study. BMJ Open 2020;10:e032268. doi:10.1136/ bmjopen-2019-032268

- Prepublication history and additional material for this paper are available online. To view these files, please visit the journal online (http://dx.doi. org/10.1136/bmjopen-2019032268).

Received 12 June 2019

Revised 05 November 2019

Accepted 11 November 2019

Check for updates

(c) Author(s) (or their employer(s)) 2020. Re-use permitted under CC BY-NC. No commercial re-use. See rights and permissions. Published by BMJ.

${ }^{1}$ Radiology, The First Affiliated Hospital of Jinan University,

Guangzhou, China

${ }^{2}$ Molecular Imaging Institute, The First Affiliated Hospital of Jinan University, Guangzhou, China

${ }^{3}$ Nuclear Medicine, The First Affiliated Hospital of Jinan University, Guangzhou, China

Correspondence to

Professor Hao Xu;

txh@jnu.edu.cn

\section{ABSTRACT}

Objectives This study was an attempt to investigate the variation trend of body composition with ageing and explore the association between regional body composition and bone mineral density (BMD).

Design Cross-sectional study.

Setting and participants A total of 5749 healthy adults aged 20-95 years was recruited from 2004 to 2017. Primary outcome measures Whole-body lean mass (LM), fat mass (FM), android FM, gynoid FM, appendicular lean mass (ALM) and BMD in the lumbar spine, femoral neck and total hip were obtained by dual-energy X-ray absorptiometry (DXA). The android/gynoid fat mass ratio (A/G FMR) based on DXA scan was calculated as an indicator of adipose distribution. Pearson correlation and multiple linear regression analyses were used to determine the associations between body composition, adipose distribution, and BMD of each skeletal site.

Results Whole-body FM, percentage of whole-body FM, Android FM and A/G FMR consistently increased with age in both genders, especially in women, and ALM began to decrease in the fifth decade for both men and women. In multivariable linear regression models with age, body mass index, A/G FMR and ALM as predictor variables, ALM was associated with the most BMD variance of all skeletal sites in men (standard $\beta$ ranged from 0.207 to 0.405 , $p<0.001$ ), although not the largest but still a positive predictor of BMD in women (standard $\beta$ ranged from 0.074 to $0.186, p<0.05)$. A/G FMR was an inverse predictor of BMD at all skeletal sites for women (standard $\beta$ ranged from -249 to $-0.052, p<0.01$ ) but not in men.

Conclusions In this large cohort of Chinese adults, ALM had a strong positive association with BMD in both genders. A/G FMR as an indicator of central adipose accumulation was inversely associated with BMD in women but not in men.

\section{INTRODUCTION}

Body weight is one of the main determinants of bone mass. It is known to be positively correlated with bone mineral density (BMD) and can partly reflect bone health. Body
Strengths and limitations of this study

- This is the first study to analyse the relationships of regional body composition (muscle and fat distribution) with bone mineral density at multiple sites in different ages and menopausal status in a large population-based sample of adults in southern China.

- A limitation of this study is that we did not obtain the actual hormone and cytokine levels, dietary intake and physical activity, which may influence bone nutrition and metabolism.

- And we only collected cross-sectional data and cannot directly conclude the causality limited by its study design.

mass index (BMI) has been widely used in epidemiological studies and clinical practice to provide a quick assessment of nutritional status and showed a positive relationship with BMD. ${ }^{1}$ Body mass is composed of lean mass (LM), fat mass (FM) and bone mass. LM is linked to significant health consequences, studied mostly in the context of severe muscle depletion (sarcopenia) that occurs with ageing and catabolic conditions. ${ }^{2}$ Moreover, studies indicate that LM may produce a positive effect on bone mass in both genders. ${ }^{3} \mathrm{FM}$ has also been shown to be a key predictor of BMD and may affect bone via both loading and hormonal mechanisms. ${ }^{4}$ Ageing is associated with gradual changes in body composition, and these changes may be entirely different between men and women, as well as premenopausal and postmenopausal women. ${ }^{56}$ To further explore the changes in body composition with age is one of the purposes of our study.

Regional body composition changes occur with age, typically characterised by decreases 
in appendicular LM (ALM) and increases in central FM. Some studies have reported that ALM and central FM may affect bone formation independent of the amount of total body composition, but the results were inconsistent. ${ }^{7-9}$ Blain $e t a l^{7}$ showed that ALM was the most influential factor contributing to BMD of the femoral neck in men, and low ALM (sarcopenia) was considered an independent risk for low BMD (osteoporosis). In contrast, Walsh $e t a l^{10}$ showed that ALM was not significantly related to BMD after adjusting body weight and physical activity in women.

Android fat represents the visceral (central) adipose tissue while gynoid fat reflects the subcutaneous (appendicular) adipose tissue. ${ }^{11}$ As the measurements of central FM used different methods or indexes, the findings regarding the relationship of central adipose tissue with BMD are more controversial. Several studies indicate that central adipose accumulation is negatively related to BMD. ${ }^{12}{ }^{13}$ On the contrary, some studies show visceral fat is positively associated with BMD in postmenopausal women. ${ }^{14}$ These heterogeneous findings may result from the rather complex mechanisms underlying the relationship between fat and bone, including mechanical loading as well as the hormones and cytokines from adipose tissue, which can indirectly influence bone metabolism to a certain extent. Moreover, gender, age, menopausal status and skeletal site differences in the relationship between BMD with ALM and central FM have not been well studied.

To our knowledge, only a few other studies have documented the relationships of ALM and android/gynoid fat mass ratio (A/G FMR) with BMD at multiple sites in different ages, menopausal status, and in both genders. In the current study, we aim to investigate the relationship between BMD and body composition, especially the effect of regional body composition on BMD. We also want to examine whether these relationships differ by gender, age and menopausal status in a large populationbased sample of Chinese adults.

\section{METHODS}

\section{Subjects}

The present study included healthy Chinese men and women aged 20-95 years old. The participants were recruited from the body composition and osteoporosis study at the First Affiliated Hospital of Jinan University (Guangzhou, China) from 2004 to 2017. Inclusion criteria for the study were Chinese individuals who appeared to be in good health and functionally independent. Subjects were excluded if they met any of the following criteria: (A) A history of fracture. (B) Medication known to affect the musculoskeletal system (antiosteoporotic drugs, androgens or antiandrogen drugs, corticosteroids, etc). (C) Chronic disease known to affect bone metabolism (hyperthyroidism, hyperparathyroidism, rheumatoid arthritis, chronic renal insufficiency, etc). (D) Metal implants (pacemakers, joint replacement device, etc).
(E) Inability to determine the menstruation state or nonnatural menopause (natural menopause was designated if there was a complete natural cessation of menses for more than 12 months). The inclusion and exclusion criteria flow chart of the study is shown in figure 1. And the current smoking and drinking situation has also been recorded. In the end, 1703 men and 4046 women were included in our study.

\section{Anthropometry, BMD and body composition measurement}

A research physician obtained information on medical history, medication use, smoking and alcohol history in a personal interview. Height and body weight were obtained based on standard methods; height was measured without shoes to the nearest $0.1 \mathrm{~cm}$, weight with only light clothing to the nearest $0.1 \mathrm{~kg}$. BMI was calculated as body weight divided by height squared $\left(\mathrm{kg} / \mathrm{m}^{2}\right)$. Subjects underwent dual-energy X-ray absorptiometry (DXA; software V.enCORE10.50.086; GE-lunar Prodigy, Wisconsin, USA) scans to measure the whole body, lumbar spine, femoral neck and total hip BMD. Total and regional LM and FM were obtained through whole body scans. Android and gynoid regions were automatically attained using the software provided by the manufacturer. The android region is defined as the portion of the abdomen included between the line joining the two superior iliac crests, extending cranially up to $20 \%$ of the distance between this line and the chin. The gynoid region is defined as the portion of the legs from the femoral greater trochanter, directed caudally up to twice the height of the android region. The appendicular region is defined as the areas including both the left and right arms and legs. Daily quality assurance scans were performed by scanning the spine phantom according to the manufacturer's instructions; the same trained technologist conducted all DXA measurements throughout the study. The coefficient of variation was less than $2 \%$ for total LM, FM, total, lumbar spine, femoral neck and total hip BMD, and less than 3\% for regional (trunk, appendicular, android and gynoid) LM and FM, and was determined by duplicate scans with repositioning between each measurement in 30 volunteer subjects.

\section{Statistical analyses}

Subjects were categorised into four groups according to gender, age and menopausal status $(786$ men $<50$ years, 917 men $\geq 50$ years, 1534 premenopausal women and 2512 postmenopausal women). The values of continuous variables were presented as the mean $\pm \mathrm{SD}$. Unpaired sample $t$-tests were used to evaluate the mean differences between different groups, and Pearson's correlation coefficients $(r)$ were used to determine the linear relationships among various parameters. We performed linear regressions to assess the association strength between ALM, A/G FMR and BMD. In the regression models, BMD (different skeletal sites) measurements were used as dependent variables, and ALM and A/G FMR were treated as independent variables; age, BMI and lifestyle 


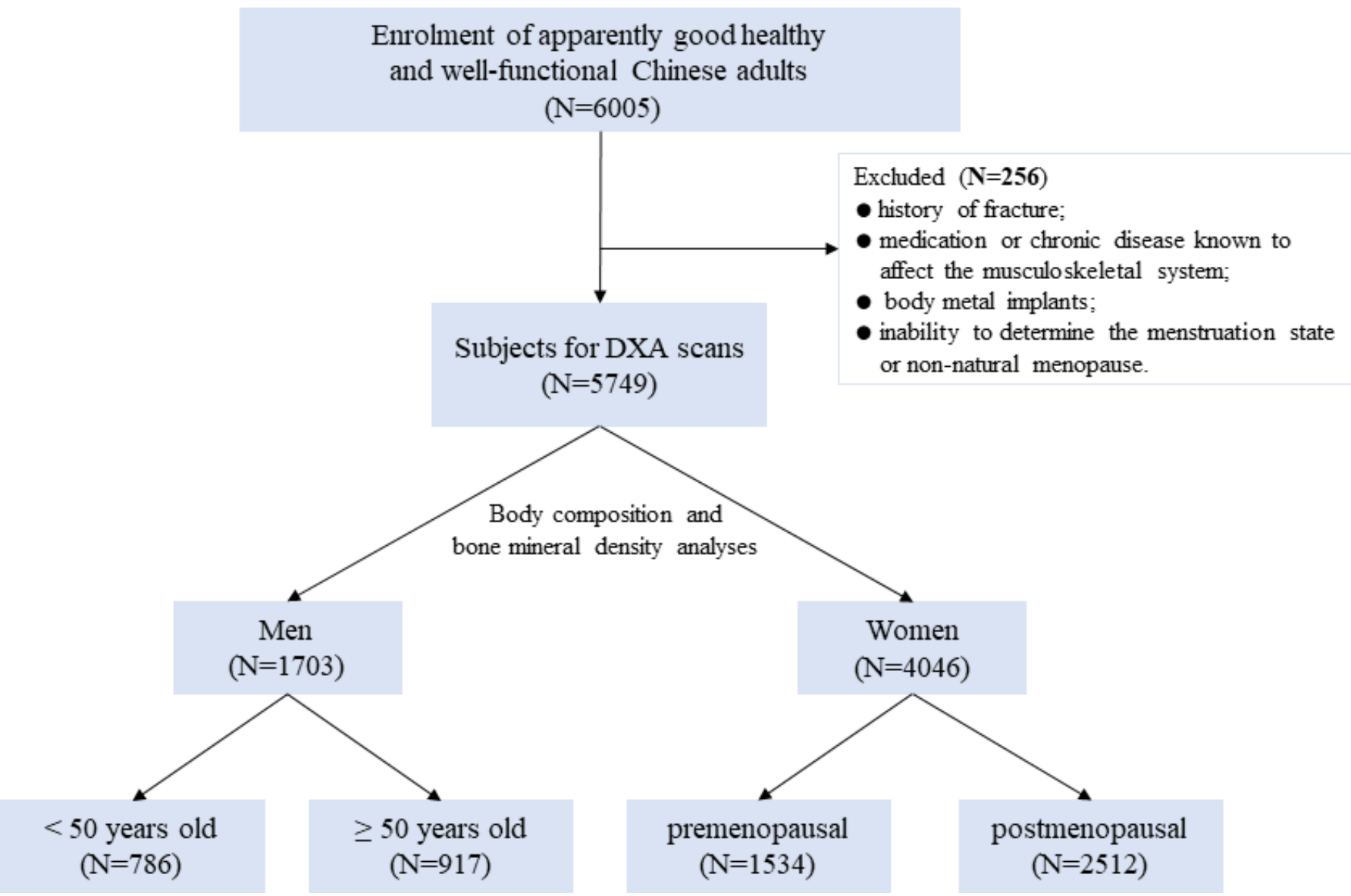

Figure 1 The study inclusion/exclusion criteria flow chart. DXA, dual-energy X-ray absorptiometry.

factors (smoking and alcohol history) were also included. When we performed linear regression analyses, we chose the enter methods and expressed the results as standard $\beta$ coefficients. All tests were two-sided, and $\mathrm{p}<0.05$ was considered statistically significant. All statistical analyses were performed using the statistical package for social sciences (V.19.0) (SPSS, Chicago, Illinois, USA).

\section{Patient and public involvement}

There was no patient or public involvement in this study.

\section{RESULTS}

\section{Basal characteristics of subjects}

Table 1 details the subject characteristics in each group. Men $\geq 50$ years had higher BMI, whole-body FM, percentage of whole-body FM (fat\%), trunk FM and appendicular FM (AFM), but lower weight and lumbar spine BMD than men < 50 years $(\mathrm{p}<0.001)$. Postmenopausal women had higher values for whole-body FM, whole-body LM, trunk FM, AFM, and A/G FMR, and lower BMD at each site than premenopausal women $(\mathrm{p}<0.05)$.

\section{Changes of BMD and body composition with age}

To further explore the distribution characteristics of body composition and BMD with age, we divided the subjects into multiple subgroups and set 10 years as one subgroup. As shown in table 2 and figure 2, whole-body
FM, fat\%, android FM and A/G FMR showed a consistent increase in both genders. Overall, the highest values of whole-body, lumbar spine, femoral neck and total hip BMD were observed in the second decade and then decreased slightly with age in men. BMD at each skeletal site increased steadily and reached a peak until perimenopause in the fourth decade in women, and then decreased dramatically after menopause. Interestingly, ALM started to decline after 50 years of age in both men and women.

\section{Associations of BMD with whole and regional body composition in different groups}

In Pearson's correlation analyses, significant positive correlations were found between BMD at all sites with height, weight, BMI, whole-body LM, whole-body FM, fat\%, android FM, gynoid FM and ALM in all groups $(r=0.218-0.616, \quad \mathrm{p}<0.05)$. A/G FMR showed positive correlations with whole-body, lumbar spine, femoral neck and total hip BMDs in men $<50$ years and $\geq 50$ years old $(\mathrm{r}=0.089 \sim 0.318, \mathrm{p}<0.001)$. However, A/G FMR was negatively correlated with whole-body and femoral neck BMDs in postmenopausal women, though the correlation strengths were weak $(r=-0.075, p<0.001$ and $r=-0.091$, $\mathrm{p}<0.001$, respectively). A/G FMR was insignificantly correlated with BMD in premenopausal women (shown in online supplementary tables 1-4). 
Table 1 Baseline characteristics of subjects

\begin{tabular}{|c|c|c|c|c|}
\hline & Male & & Female & \\
\hline & Age $<50$ years & Age $\geq 50$ years & Premenopausal & Postmenopausal \\
\hline No. of subjects & 786 & 917 & 1534 & 2512 \\
\hline Age (years) & $36.8 \pm 8.7$ & $65.8 \pm 10.0^{*}$ & $37.4 \pm 8.7$ & $63.9 \pm 9.1^{*}$ \\
\hline Weight (kg) & $63.9 \pm 12.4$ & $63.8 \pm 10.8^{*}$ & $52.7 \pm 9.1$ & $55.0 \pm 8.9$ \\
\hline Height (cm) & $168.8 \pm 5.8$ & $166.7 \pm 6.1$ & $158.0 \pm 4.9$ & $155.9 \pm 5.3^{*}$ \\
\hline $\mathrm{BMI}\left(\mathrm{kg} / \mathrm{m}^{2}\right)$ & $22.4 \pm 3.9$ & $22.9 \pm 3.3^{*}$ & $21.1 \pm 3.3$ & $22.6 \pm 3.3$ \\
\hline Body composition measu & & & & \\
\hline Whole body FM & $13.7 \pm 7.6$ & $15.2 \pm 6.5^{\star}$ & $16.7 \pm 6.0$ & $18.9 \pm 6.2 \dagger$ \\
\hline Whole body LM & $47.6 \pm 6.0$ & $46.1 \pm 5.9$ & $33.8 \pm 4.3$ & $34.2 \pm 4.0 \ddagger$ \\
\hline Fat $\%$ & $20.2 \pm 8.3$ & $23.0 \pm 7.3^{*}$ & $30.1 \pm 6.7$ & $33.6 \pm 6.8$ \\
\hline Trunk FM & $8.2 \pm 5.0$ & $9.3 \pm 4.4^{*}$ & $8.7 \pm 3.6$ & $10.6 \pm 3.7 \dagger$ \\
\hline Trunk LM & $22.4 \pm 3.0$ & $22.4 \pm 2.9$ & $16.4 \pm 2.2$ & $17.1 \pm 2.1$ \\
\hline Appendicular FM & $5.0 \pm 2.6$ & $5.3 \pm 2.1^{*}$ & $7.3 \pm 2.5$ & $7.5 \pm 2.6^{*}$ \\
\hline Appendicular LM & $21.4 \pm 3.2$ & $19.9 \pm 3.1$ & $14.4 \pm 2.2$ & $14.0 \pm 2.1$ \\
\hline A/G FMR & $0.57 \pm 0.17$ & $0.69 \pm 0.19$ & $0.42 \pm 0.10$ & $0.56 \pm 0.15^{\star}$ \\
\hline Bone mineral density $(\mathrm{g} / \mathrm{c}$ & & & & \\
\hline Whole body & $1.105 \pm 0.109$ & $1.109 \pm 0.105$ & $1.085 \pm 0.094$ & $0.994 \pm 0.106^{\star}$ \\
\hline Lumbar spine & $1.114 \pm 0.162$ & $1.099 \pm 0.200^{\star}$ & $1.124 \pm 0.155$ & $0.950 \pm 0.186^{\star}$ \\
\hline Femoral neck & $0.921 \pm 0.140$ & $0.839 \pm 0.140$ & $0.907 \pm 0.122$ & $0.760 \pm 0.132 \dagger$ \\
\hline Total hip & $0.941 \pm 0.150$ & $0.911 \pm 0.147$ & $0.942 \pm 0.132$ & $0.817 \pm 0.144 \dagger$ \\
\hline Current smoker (\%) & $\mathrm{n}=141(17.9 \%)$ & $\mathrm{n}=119(13.0 \%)$ & $\mathrm{n}=37(2.4 \%)$ & $\mathrm{n}=70(2.8 \%)$ \\
\hline Current alcohol user (\%) & $\mathrm{n}=121(15.4 \%)$ & $\mathrm{n}=117(12.8 \%)$ & $\mathrm{n}=35(2.3 \%)$ & $\mathrm{n}=38(1.5 \%)$ \\
\hline
\end{tabular}

Values are presented as number, mean \pm SD or percentage.

Compared with the same gender of the different age group (unpaired-sample t-tests or $\chi^{2}$ test).

$P$ value was determined by the unpaired-sample t-tests.

${ }^{*} P<0.001$.

$+P<0.01$.

$\ddagger P<0.05$.

A/G FMR, android/gynoid fat mass ratio; BMI, body mass index; fat\%, percentage of whole body fat mass; FM, fat mass; LM, lean mass.

To further explore the independent predictive value of $\mathrm{A} / \mathrm{G}$ FMR and ALM for BMD at all sites in every group, covariates such as age, BMI, smoking and alcohol consumption associated with BMD were also included in the multiple linear regression analyses (shown in tables 3 and 4). Overall, the significant variables accounted for $10.7 \sim 37.4 \%$ of the variability in BMD. Taking into consideration the dynamic change of BMD between the period of bone modelling (20-29 years) and that of bone remodelling (30-50 years), and the subjects aged 20-29 years were excluded in the regression analyses. The effect sizes of A/G FMR and ALM on BMD were different according to the skeletal site and age group. A/G FMR had inverse associations with whole-body, lumbar spine, femoral neck and total hip BMDs in both premenopausal (standard $\beta$ from -0.249 to $-0.155, p<0.001$ ) and postmenopausal (standard $\beta$ from -0.135 to $-0.052, \mathrm{p}<0.01$ ) women, while men had no significant relationship between A/G FMR and each part of BMD after adjustment. ALM was positively associated with whole-body, lumbar spine, femoral neck and total hip BMDs in both men and women, and the correlations were higher in men than in women (standard $\beta$ from 0.309 to 0.405 of men $<50$ years vs standard $\beta$ from 0.074 to 0.179 of premenopausal women, $p<0.001$; standard $\beta$ from 0.207 to 0.321 of men $\geq 50$ years vs standard $\beta$ from 0.152 to 0.186 of postmenopausal women, $\mathrm{p}<0.001)$.

\section{DISCUSSION}

The global epidemic of obesity has become a significant concern in our daily life as it has a close relationship with cardiovascular and cerebrovascular diseases and influences bone health. ${ }^{15}$ As a part of body composition, bone density was mainly determined by body weight and BMI. Low BMI had been regarded as a risk factor for osteoporotic hip fracture in both men and women. ${ }^{16}$ In this study, we investigated a wide range of healthy Chinese adults aged 20-95 years to further explore the factors that may influence bone health. We divided the participants into 


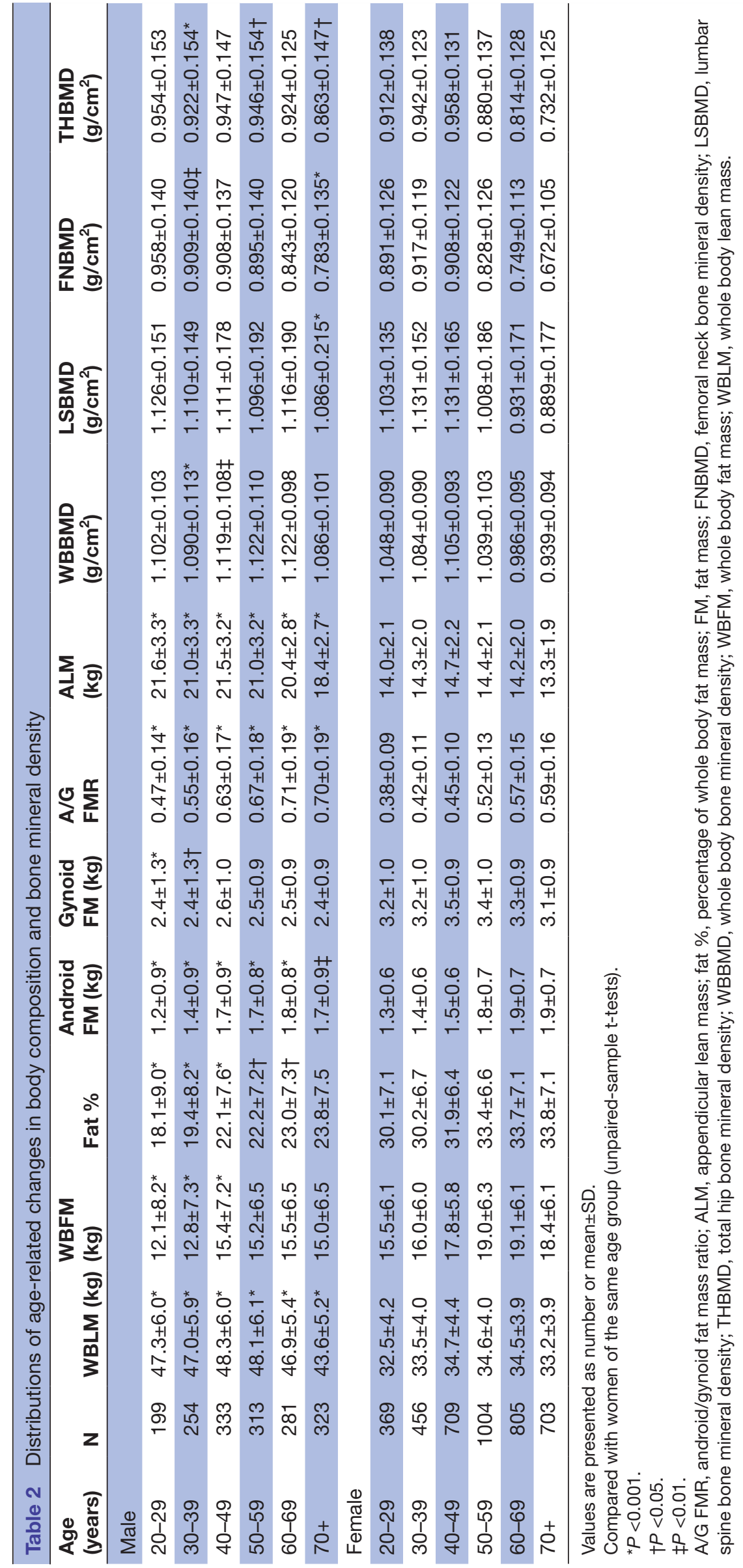




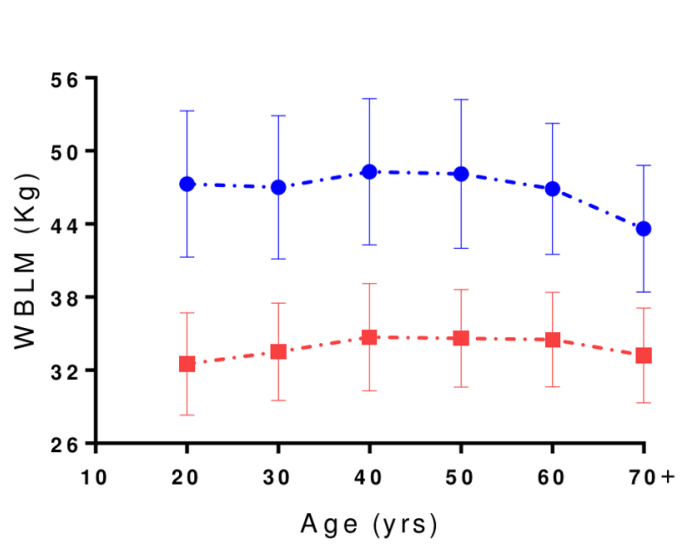

-O-Males
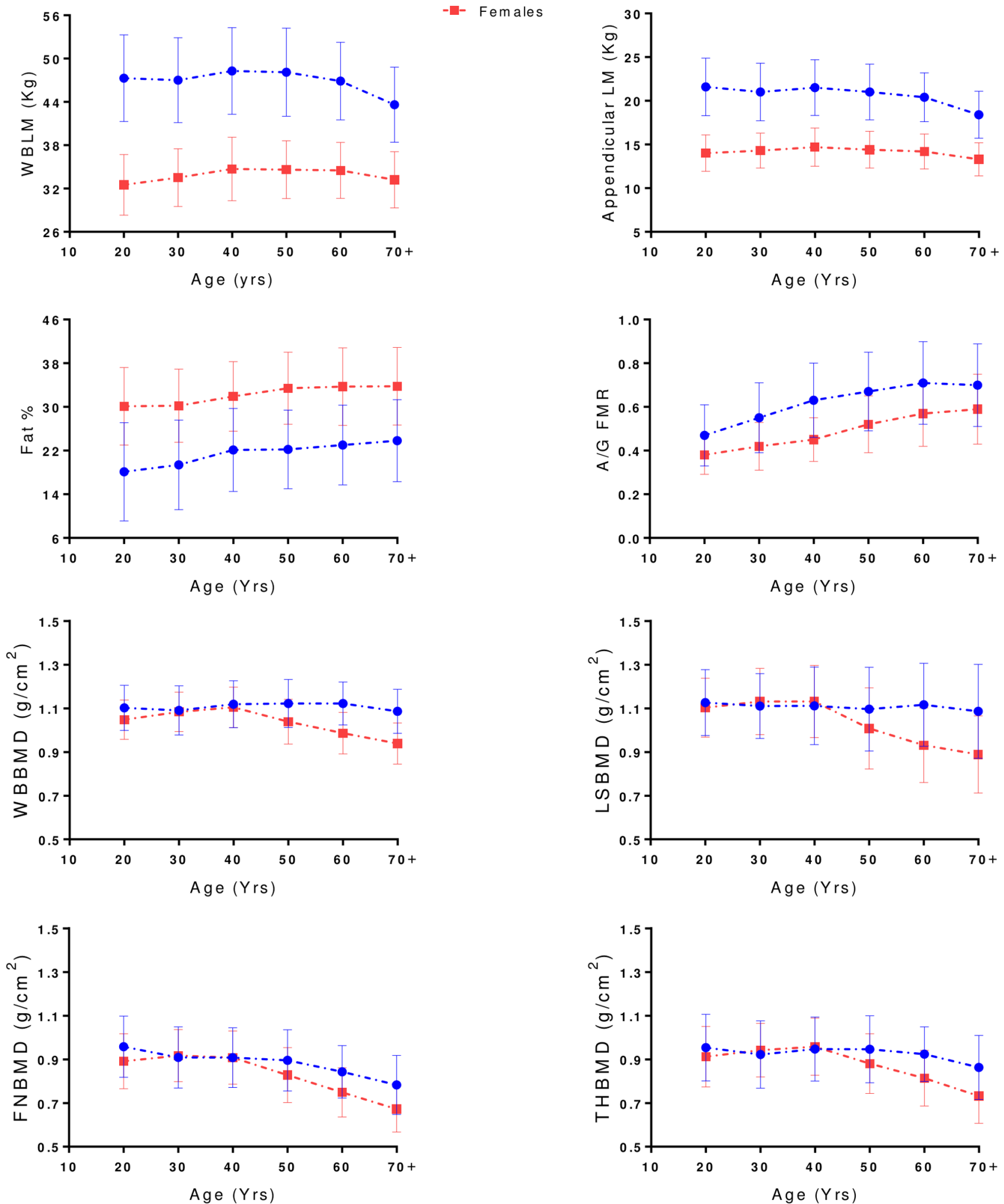

Figure 2 The age-related change in whole-body lean mass (WBLM), appendicular lean mass (ALM), percentage of wholebody fat mass (fat\%), A/G FMR and bone mineral density (BMD) in men and women. A/G FMR, android/gynoid fat mass ratio; FNBMD, femoral neck bone mineral density; LSBMD, lumbar spine bone mineral density; THBMD, total hip bone mineral density; WBBMD, whole-body bone mineral density.

different age groups to investigate the changes in body composition and BMD with age in men and women. We also performed multivariable regression analyses to confirm A/G FMR and ALM were independent predictors for BMD after adjusting for age, BMI, smoking and alcohol consumption. The current report provided more 


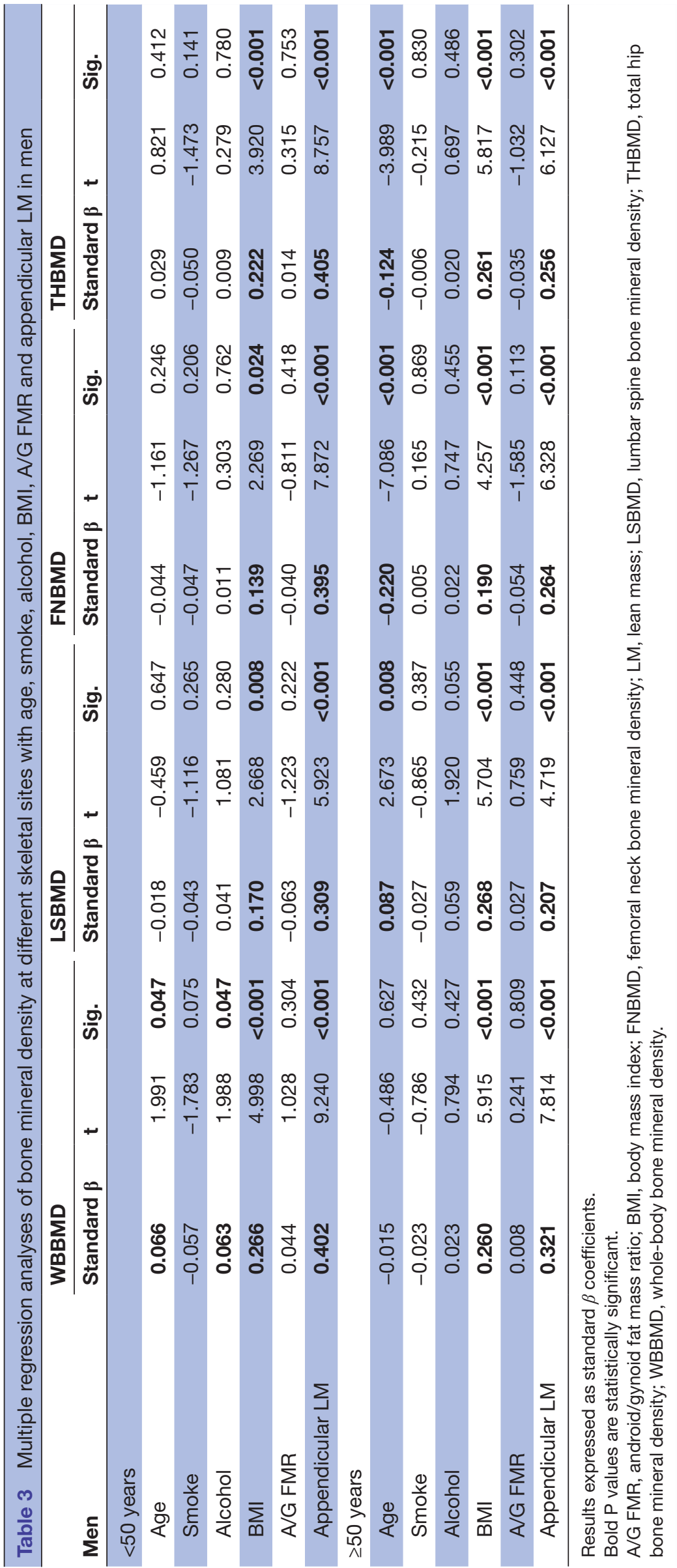




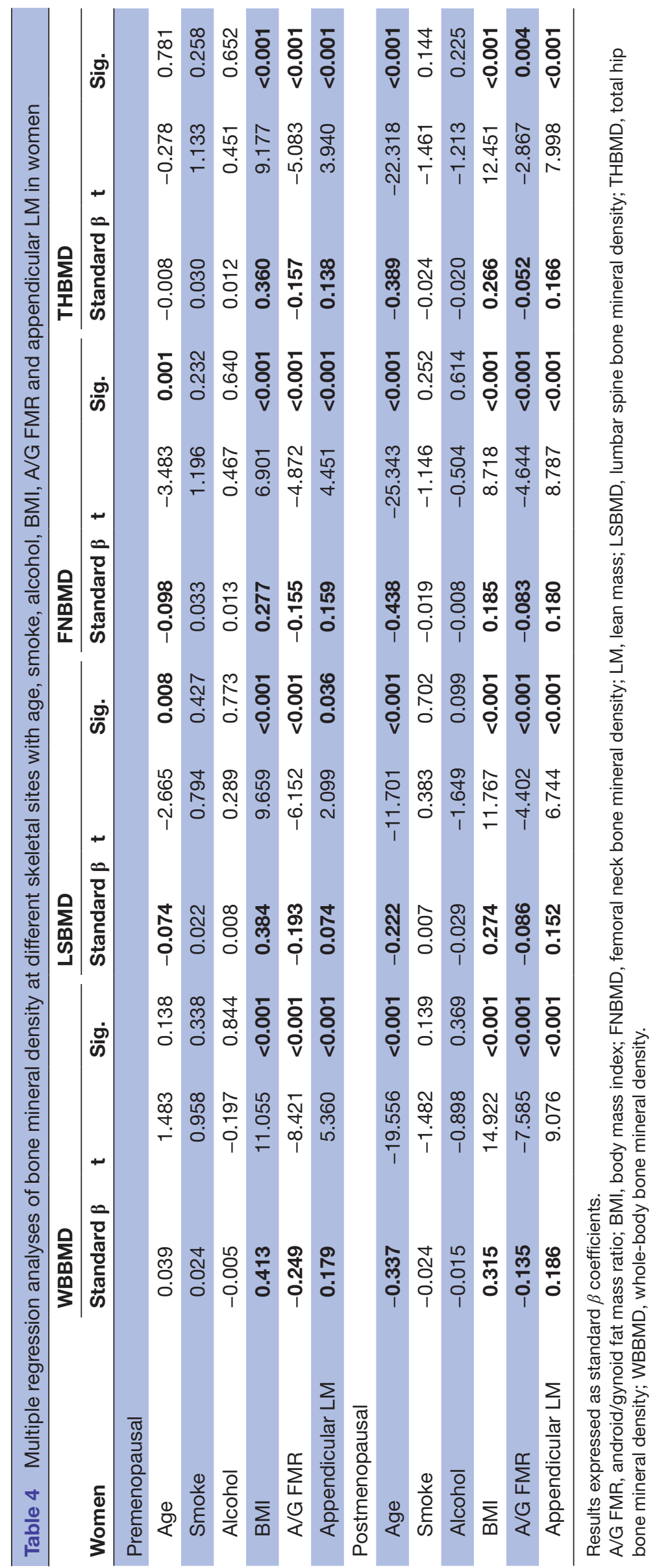


detailed and impressive results which were different from previous studies performed in America ${ }^{13}$ and Australia. ${ }^{1}$ We found that ALM positively correlated with BMD in both genders, and low ALM was related to low BMD. A/G FMR as an indicator of central adipose accumulation was inversely associated with BMD in women but not in men. These results, based on a large population of Chinese adults, were convincing.

In this study, we found that whole-body LM reached a peak level in the 40-49 years age group, and then decreased gradually in both genders, whereas whole-body FM steadily increased from youth to older age in both genders. After analysing the baseline characteristics, we found that the decreased whole-body LM in older men was primarily due to a decrease in ALM. Moreover, the increased whole-body FM in older men and women mainly arose from the increase of trunk FM. In the meantime, we found that $A / G$ FMR increased with age in both men and women. Men had whole-body, lumbar spine, femoral neck and total hip BMD peaks earlier than women. BMD at all sites decreased slightly in older men, but more obviously in postmenopausal women. These results showed a healthy bone mass for each age group in both men and women. The BMD at each region lower than the reference ranges in the respective age groups should alarm the physician for appropriate intervention.

In our study, the results showed that both total LM and total FM are positively associated with BMD in both genders. The effect sizes of total LM and total FM to BMD were different according to gender, menopausal status and age. Total LM is a stronger protective factor to BMD at all sites in men and premenopausal women. Total FM is a stronger contributor to BMD at all sites in postmenopausal women. Several potential theories may explain the observed findings. The influences of LM on BMD may attribute to the direct mechanical effects of muscle, which produces a positive osteogenic response to bone formation. On one hand, whole-body LM, which accounted for a large proportion of body weight in both men and women, would have the effect of a gravitational loading on the bone. On the other hand, the contraction strength of lean muscle should also be considered a specific mechanism of action. A previous study reported that the augmentation and thickening of bone trabecula was an adaption to increased mechanical stress. However, whole-body FM only accounted for a small proportion of body weight in both men and women, but it still had a significant and positive correlation with BMD, especially in postmenopausal women, in whom a higher standard $\beta$ value with BMD in all the skeletal sites was shown compared with whole-body LM. Several mechanisms could explain the association between fat tissue and BMD. The outcomes of fat acting on the bone may be influenced by weight-bearing and by non-weight-bearing effects, including the hormonal metabolism of adipocytes. We speculate that this fat-related mechanism may help to interpret this finding, as the postmenopausal women also had the highest whole-body FM, more than men and premenopausal women in our study. Several hormones, including insulin, leptin, adiponectin and adipocytic oestrogens, were found to be secreted from adipose tissue, which can influence bone metabolism through the endocrine pathway. Also, the enzyme aromatase in adipose tissue can convert androgen to oestrogen and result in an elevated oestrogen level. These bone protective hormones lead to a positive influence on bone formation via stimulating the differentiation of osteoblasts and preventing osteoclast-mediated bone resorption. This finding further confirmed the results from previous studies that FM should have a positive relationship with bone mass. ${ }^{817}$

Though whole-body LM and FM were found to be positively associated with BMD in both genders, how regional body compositions and differences in fat distribution influence bone metabolism aroused our curiosity. To investigate the effect of ALM and A/G FMR on BMD at various ages, factors including gender, age, BMI, smoking and alcohol consumption, which may have close relationships with BMD, were considered in multiple linear regression analyses. In the current study, we found that ALM was positively related to BMD at all sites after adjustment for BMI and age in both genders. ALM is considered one of the most important indexes of the diagnostic criterion for sarcopenia. ${ }^{18} 19$ A study of 679 men aged 40-79 years suggested low ALM was associated with low BMD (whole body, femoral neck, total hip and lumbar spine) and osteoporosis independent of age, height, physical activity and other lifestyles. ${ }^{20}$ Blain $e t a l^{7}$ also found that ALM was most strongly associated with femoral neck BMD independent of nutritional and hormonal factors, and other lifestyles in men. There are several mechanisms that may explain the observed association between ALM and BMD. The amount of ALM was smaller than trunk LM in this study, suggesting ALM may affect the bone via contraction strength instead of gravitational loading, especially in men younger than 50 years, in whom the strongest relationships with BMD in all sites were demonstrated, compared with other groups. Systemic factors that simultaneously involve both ALM and bone metabolism may exist as the decline of ALM was almost parallel with BMD in both genders. For example, the hormone oestrogen can strengthen the synthesis of muscle protein and promote calcium deposition in bone tissue, which leads to an increased LM and BMD concurrently. ${ }^{21}$ However, a prospective study is needed to explore the potential mechanism further.

Depot-specific fat has been known to play a different role in terms of obesity and metabolism. A previous study indicated that different fat depots might have distinct relationships with bone mass; ${ }^{22}$ Marques et al reported AFM had a positive association with femoral neck BMD in older women. ${ }^{23}$ Inconsistent with that, several studies stated AFM had no $^{24}$ or a negative ${ }^{25}$ relationship with BMD. Freitas $e t a l^{26}$ showed that central fat was positively associated with BMD and regarded as an independent and protective factor for the presence of osteoporosis or 
osteopenia. Sharma $e t a l^{27}$ reported that a bigger trunk (central) FM was associated with increased BMD in the total hip and femoral neck, regardless of HIV status in women. Fat distribution differences seem to produce a meaningful but contradictory effect on bone mass based on previous studies. To further confirm this finding, we performed Pearson's correlation to assess the relationship between android FM, gynoid FM and each part of BMD with a large sample size. The results suggested that both android FM and gynoid FM positively correlated with BMD in all men and women, which was partly consistent with previous studies. ${ }^{22}{ }^{24}$ In contrast, some studies reported abdominal fat and android fat measured by CT or DXA had a negative association with BMD after adjusting for total LM or BMI, suggesting central fat deposition was not beneficial for bone. ${ }^{13}$ Surprisingly, we found that $\mathrm{A} / \mathrm{G}$ FMR showed a diverse correlation with BMD in men and women. In the multiple regression analysis, A/G FMR was an inverse predictor of BMD at all skeletal sites for females (standard $\beta$ ranged from -249 to $-0.052, \mathrm{P}<0.01)$ but not in males. Kim $e t a t^{22}$ also reported that A/G FMR was inversely associated with the trabecular bone score after age adjustment $(\mathrm{r}=-0.288$, $\mathrm{p}<0.05$ ), which was similar to our findings. Android fat mainly represents visceral fat in the epigastric region, while gynoid fat reflects peripheral (or subcutaneous) fat in the leg. Higher A/G FMR indicating higher visceral fat or lower peripheral fat revealed that subjects with higher A/G FMR had lower BMD in postmenopausal women, whose ovarian hormones tend to be depleted and lead to a higher subcutaneous lipoprotein lipase activity ratio and predominant fat storage in visceral fat depots. ${ }^{28}$ Kim $e t a l^{29}$ found that visceral fat has a negative association among postmenopausal Korean women with lumbar spine BMD after adjustment for weight. Zhu et al reported that a trunk-to-limb FM ratio (a surrogate of visceral fat) had a negative association with total body bone mass in young adults. ${ }^{17}$ Gilsanz et $a l^{30}$ suggested that subcutaneous and visceral fat had opposite effects on femoral bone structure and strength in healthy young women, and proposed that subcutaneous fat may be beneficial to the bone, whereas visceral fat may have a negative association with the bone. These heterogeneous findings may be related to the use of diverse methods of measuring body composition and BMD, disparities in study design, or the different criteria for group division. Moreover, the inconsistency of findings may be due to the rather complex mechanisms underlying the relationship between fat and bone. As a result, we subdivided Chinese people into different age and gender groups and found that android fat increased with age, especially in women, whose android fat accounted for a more substantial proportion of body weight and had a stronger relationship with BMD in our study. To avoid multicollinearity, we included $\mathrm{A} / \mathrm{G}$ FMR instead of the total and regional body composition into the same multiple regression analysis when we explored the associations of regional fat depots with BMD, which provided a more reliable result.

\section{Limitations}

This study had several limitations. First, we did not obtain a blood sample from participants; thus, the actual hormone and cytokine levels were unknown. The potential mechanisms acting on bone mass mainly referenced previous reports based on our data and the statistical results. Second, we only collected cross-sectional data and cannot directly conclude the causality limited by its study design. Third, though we evaluated the relationships of body composition and fat distribution with BMD by adjusting age, BMI, smoking and alcohol consumption, other confounders such as socioeconomic status, dietary intake and physical activity, which may influence bone nutrition and metabolism, were not considered as covariates in the multivariable regression analyses. Fourth, a more substantial amount of visceral FM was found in Asians compared with the European people for a given amount of body fat. ${ }^{31}$ Therefore, ethnic differences should be considered when interpreting the findings.

\section{CONCLUSIONS}

In summary, in this large cohort of Chinese adults, ALM had a strong positive association with BMD in both genders suggesting that low ALM is related to low BMD and may be considered an independent risk factor for osteoporosis. A/G FMR, an indicator of central adipose accumulation, was inversely associated with BMD in women but not in men.

Acknowledgements The authors thank all participating subjects.

Contributors ZX, ZT, JS, YC and YT collected, analysed and interpreted data; $Z X, B G$ and JG wrote the paper. HX, the principal investigator and innovator, conceptualised, designed and revised the manuscript.

Funding This work was supported by the National Natural Science Foundation of China (Grant No. 81871383) and the Medical Scientific Research Foundation of Guangdong Province, China (Grant No. A2018132).

Competing interests None declared.

Patient consent for publication Not required.

Ethics approval The study was approved by the Ethics Committee of the First Affiliated Hospital of Jinan University.

Provenance and peer review Not commissioned; externally peer reviewed.

Data availability statement Data are available upon reasonable request.

Open access This is an open access article distributed in accordance with the Creative Commons Attribution Non Commercial (CC BY-NC 4.0) license, which permits others to distribute, remix, adapt, build upon this work non-commercially, and license their derivative works on different terms, provided the original work is properly cited, appropriate credit is given, any changes made indicated, and the use is non-commercial. See: http://creativecommons.org/licenses/by-nc/4.0/.

ORCID iD

Zeyu Xiao http://orcid.org/0000-0003-2220-5675

\section{REFERENCES}

1 Zhu K, Hunter M, James A, et al. Associations between body mass index, lean and fat body mass and bone mineral density in 
middle-aged Australians: the Busselton healthy ageing study. Bone 2015;74:146-52

2 Chen S-C, Chung W-S, Wu P-Y, et al. Associations among geriatric nutrition risk index, bone mineral density, body composition and handgrip strength in patients receiving hemodialysis. Nutrition 2019;65:6-12.

3 Stroup BM, Hansen KE, Krueger D, et al. Sex differences in body composition and bone mineral density in phenylketonuria: a crosssectional study. Mol Genet Metab Rep 2018;15:30-5.

4 Heiss CJ, Sanborn CF, Nichols DL, et al. Associations of body fat distribution, circulating sex hormones, and bone density in postmenopausal women. J Clin Endocrinol Metab 1995;80:1591-6.

5 Guo B, Wu Q, Gong J, et al. Gender difference in body fat for healthy Chinese children and adolescents. Childhood Obesity 2016;12:144-54.

6 Guo B, Wu Q, Gong J, et al. Relationships between the lean mass index and bone mass and reference values of muscular status in healthy Chinese children and adolescents. J Bone Miner Metab 2016;34:703-13.

7 Blain $\mathrm{H}$, Jaussent A, Thomas E, et al. Appendicular skeletal muscle mass is the strongest independent factor associated with femora neck bone mineral density in adult and older men. Exp Gerontol 2010;45:679-84.

8 Bogl LH, Latvala A, Kaprio J, et al. An investigation into the relationship between soft tissue body composition and bone mineral density in a young adult twin sample. J Bone Miner Res 2011;26:79-87.

9 Cui L-H, Shin M-H, Kweon S-S, et al. Relative contribution of body composition to bone mineral density at different sites in men and women of South Korea. J Bone Miner Metab 2007;25:165-71.

10 Walsh CJ, Phan CM, Misra M, et al. Women with anorexia nervosa: finite element and trabecular structure analysis by using flat-panel volume CT. Radiology 2010;257:167-74.

11 Kang SM, Yoon JW, Ahn HY, et al. Android fat depot is more closely associated with metabolic syndrome than abdominal visceral fat in elderly people. PLoS One 2011;6:e27694.

12 Choi HS, Kim KJ, Kim KM, et al. Relationship between visceral adiposity and bone mineral density in Korean adults. Calcif Tissue Int 2010;87:218-25.

13 Katzmarzyk PT, Barreira TV, Harrington DM, et al. Relationship between abdominal fat and bone mineral density in white and African American adults. Bone 2012;50:576-9.

14 Warming L, Ravn P, Christiansen C. Visceral fat is more important than peripheral fat for endometrial thickness and bone mass in healthy postmenopausal women. Am J Obstet Gynecol 2003;188:349-53.

15 Shapses SA, Pop LC, Wang Y. Obesity is a concern for bone health with aging. Nutr Res 2017;39:1-13.
16 De Laet C, Kanis JA, Odén A, et al. Body mass index as a predictor of fracture risk: a meta-analysis. Osteoporos Int 2005;16:1330-8.

17 Zhu K, Briffa K, Smith A, et al. Gender differences in the relationships between lean body mass, fat mass and peak bone mass in young adults. Osteoporos Int 2014;25:1563-70.

18 Baumgartner RN, Koehler KM, Gallagher D, et al. Epidemiology of sarcopenia among the elderly in New Mexico. Am J Epidemiol 1998;147:755-63.

19 Melton LJ, Khosla S, Riggs BL, et al. Epidemiology of sarcopenia. Mayo Clin Proc 2000;75 Suppl:S10-13.

20 Verschueren S, Gielen E, O'Neill TW, et al. Sarcopenia and its relationship with bone mineral density in middle-aged and elderly European men. Osteoporos Int 2013;24:87-98.

21 Seeman E. Clinical review 137: sexual dimorphism in skeletal size, density, and strength. J Clin Endocrinol Metab 2001;86:4576-84.

$22 \mathrm{Kim} \mathrm{JH}$, Choi HJ, Ku EJ, et al. Regional body fat depots differently affect bone microarchitecture in postmenopausal Korean women Osteoporos Int 2016;27:1161-8.

23 Marques EA, Moreira P, Wanderley F, et al. Appendicular fat mass is positively associated with femoral neck bone mineral density in older women. Menopause 2012;19:311-8.

24 Kuwahata A, Kawamura Y, Yonehara Y, et al. Non-weight-bearing effect of trunk and peripheral fat mass on bone mineral density in pre- and post-menopausal women. Maturitas 2008;60:244-7.

25 Yoo HJ, Park MS, Yang SJ, et al. The differential relationship between fat mass and bone mineral density by gender and menopausal status. J Bone Miner Metab 2012;30:47-53.

26 Freitas PMSS, Garcia Rosa ML, Gomes AM, et al. Centra and peripheral fat body mass have a protective effect on osteopenia or osteoporosis in adults and elderly? Osteoporos Int 2016;27:1659-63.

27 Sharma A, Flom PL, Rosen CJ, et al. Racial differences in bone loss and relation to menopause among HIV-infected and uninfected women. Bone 2015;77:24-30.

28 Tchernof A, Desmeules A, Richard C, et al. Ovarian hormone status and abdominal visceral adipose tissue metabolism. J Clin Endocrinol Metab 2004;89:3425-30.

29 Kim CJ, Oh KW, Rhee EJ, et al. Relationship between body composition and bone mineral density (BMD) in perimenopausal Korean women. Clin Endocrinol 2009;71:18-26.

30 Gilsanz V, Chalfant J, Mo AO, et al. Reciprocal relations of subcutaneous and visceral fat to bone structure and strength. J Clin Endocrinol Metab 2009;94:3387-93.

31 Lesser IA, Gasevic D, Lear SA. The effect of body fat distribution on ethnic differences in cardiometabolic risk factors of Chinese and Europeans. Appl Physiol Nutr Metab 2013;38:701-6. 